\title{
Lung irradiation induces pulmonary vascular remodelling resembling pulmonary arterial hypertension
}

\author{
G Ghobadi, ${ }^{1,2}$ B Bartelds, ${ }^{3}$ S J van der Veen, ${ }^{1,2}$ M G Dickinson, ${ }^{3}$ S Brandenburg, ${ }^{4}$ \\ R M F Berger, ${ }^{3}$ J A Langendijk, ${ }^{1}$ R P Coppes, ${ }^{1,2}$ P van Luijk ${ }^{1}$
}

\begin{abstract}
- An additional figure is published online only. To view this file please visit the journal online (http://thorax.bmj.com/ content/67/4.toc).

${ }^{1}$ Department of Radiation Oncology, University Medical Center Groningen, University of Groningen, Groningen, The Netherlands

2Department of Cell Biology, Section of Radiation and Stress Cell Biology, University Medical Center Groningen, University of Groningen, Groningen, The Netherlands

${ }^{3}$ Center for Congenital Heart Disease, Pediatric Cardiology, Beatrix Children Hospital, University Medical Center Groningen, University of Groningen, Groningen, The Netherlands

${ }^{4}$ Kernfysisch Versneller Instituut, University of Groningen, Groningen, The Netherlands
\end{abstract}

\section{Correspondence to}

Dr Peter van Luijk, Department of Radiation Oncology, University Medical Center Groningen, University of Groningen, P.0. Box 30001, 9700 RB Groningen, The Netherlands;

p.van.luijk@umcg.nl

Received 15 April 2011 Accepted 1 December 2011 Published Online First 26 December 2011

\section{ABSTRACT \\ Background Pulmonary arterial hypertension (PAH) is a commonly fatal pulmonary vascular disease that is often diagnosed late and is characterised by a progressive rise in pulmonary vascular resistance resulting from typical vascular remodelling. Recent data suggest that vascular damage plays an important role in the development of radiation-induced pulmonary toxicity. Therefore, the authors investigated whether irradiation of the lung also induces pulmonary hypertension.}

Methods Different sub-volumes of the rat lung were irradiated with protons known to induce different levels of pulmonary vascular damage.

Results Early loss of endothelial cells and vascular oedema were observed in the irradiation field and in shielded parts of the lung, even before the onset of clinical symptoms. 8 weeks after irradiation, irradiated volume-dependent vascular remodelling was observed, correlating perfectly with pulmonary artery pressure, right ventricle hypertrophy and pulmonary dysfunction. Conclusions The findings indicate that partial lung irradiation induces pulmonary vascular remodelling resulting from acute pulmonary endothelial cell loss and consequential pulmonary hypertension. Moreover, the close resemblance of the observed vascular remodelling with vascular lesions in PAH makes partial lung irradiation a promising new model for studying PAH.

\section{INTRODUCTION}

Pulmonary arterial hypertension (PAH) is a severe and progressive form of pulmonary hypertension that leads to right heart failure and premature death, and no cure is available. ${ }^{1} \mathrm{PAH}$ is characterised by typical angioproliferative lesions, such as neointimal lesions, leading to increased pulmonary vascular resistance. ${ }^{2}$ The elevated pulmonary vascular resistance leads to high right ventricle systolic pressure and subsequent right ventricular hypertrophy $(\mathrm{RVH})$ ultimately resulting in heart failure. $^{3}{ }^{4}$ The pathogenesis of the increased pulmonary vascular resistance is due to a combination of sustained vasoconstriction, arterial wall remodelling and thrombosis. ${ }^{5}$ Although the typical histopathology of the angioproliferative lesions is well described, the mechanisms are so far unknown. A variety of animal models have been developed to study the mechanism of development and maintenance of pulmonary hypertension. However, few of these models fully describe human

\section{Key messages}

What is the key question?

- Can lung irradiation be used as a new model for studying pulmonary arterial hypertension (PAH)?

What is the bottom line?

- Radiation-induced vascular remodelling leads to pulmonary hypertension with features closely resembling PAH.

Why read on?

- Partial lung irradiation is a promising new model to study and develop strategies for the prevention and treatment of pulmonary arterial hypertension.

$\mathrm{PAH}^{6}$ Interestingly, we recently showed in a preclinical model that thoracic irradiation also leads to vascular damage, which is the predominant histological change after irradiation of large volumes with a relatively low dose. ${ }^{7}$ Our findings agreed with suggestions from clinical ${ }^{8}$ and preclinical studies, ${ }^{9}$ indicating that treatment of large lung volumes even at a low dose is an important risk factor in the development of pulmonary toxicity. Moreover, whole thoracic irradiation with a sublethal dose was shown to induce increased pulmonary vascular resistance and a decrease in pulmonary arterial distensibility and vascular density early after radiation. ${ }^{10}$ These features seem similar to those observed in patients with pulmonary hypertension. ${ }^{11}$ Interestingly, patients already suffering from pre-existing pulmonary vascular disease, manifesting as subclinical increases in pulmonary artery pressure, are known to have an increased risk for radiation-induced pulmonary toxicity. ${ }^{12}$ However, the type, evolution and consequences of pulmonary vascular remodelling after radiation are largely unknown. In the present study, we hypothesise that vascular damage after irradiation of the lung may develop into PAH. Using high-precision proton radiation beams, this hypothesis was tested by inducing different levels of vascular damage by irradiating small, intermediate and large volumes of rat lungs with graded radiation doses, resulting in an equal risk of inducing pulmonary dysfunction. ${ }^{13}$ Since vascular damage plays a central role in radiation- 
induced pulmonary dysfunction and pulmonary hypertension, we investigated the commonalities between radiation-induced vascular remodelling and previously described PAH models.

\section{MATERIAL AND METHODS \\ Animals}

Adult male albino Wistar rats $(\mathrm{n}=78,270-320 \mathrm{~g}, 8-9$ weeks old) of the Hsd/Cpb:WU strain bred in a specific pathogen-free colony (Harlan-CPB, Rijswijk, The Netherlands) were used in the experiments. They were housed five to a cage under a $12 \mathrm{~h}$ light $-12 \mathrm{~h}$ dark cycle and fed rodent chow (RMH-B, Hope Farms, Woerden, The Netherlands) and water ad libitum. The experiments were performed in agreement with the Netherlands Experiments on Animals Act (1977) and the European Convention for the Protection of Vertebrate Animals Used for Experimental Purposes (Strasbourg, 18.III.1986).

\section{Irradiation technique}

To induce different levels of vascular damage, $33 \%, 50 \%, 75 \%$ or $100 \%$ of the rats' lungs were irradiated to $28,20 / 22,17$ and 13 Gy (single fraction) respectively (figure 1). This was done with $150 \mathrm{MeV}$ protons from the cyclotron at the Kernfysisch Versneller Instituut, using the shoot-through technique as published previously. ${ }^{13-16}$ This results in very sharp lateral field edges $(20-80 \% \text { isodose distance: } 1 \mathrm{~mm})^{16}$ thus sparing the shielded part of the lung very effectively. The irradiation ports (figure 1) were designed using CT scans of animals of the same age and weight. ${ }^{17}$

\section{Histopathology}

For morphological analysis, tissue samples were taken from infield and out-of-field of the left lung at a distance of at least $3 \mathrm{~mm}$ from the field edge and compared with non-irradiated controls $(n=3)$. The radiation response of the pulmonary vasculature was assessed by evaluating the morphology before ( 2 weeks post irradiation) and at the peak of pulmonary dysfunction ( 8 weeks post irradiation) ${ }^{17}$ in three rats per group. Pulmonary sections (5 $\mu \mathrm{m}$ thick) were stained with haematoxylin and eosin, rat endothelial cell (EC) specific marker (HIS52) or Verhoeff's elastica stain. Since $70 \%$ of the pulmonary vascular bed consists of radiosensitive microvasculature ${ }^{18} 19$ and damage of these can be expected to have the largest impact on lung function, small intra-acinar vessels $(<50 \mu \mathrm{m})$ were selected for morphological analysis. For morphometric analysis of the vascular dimensions, lung sections with Verhoeff's elastica stain were used according to van Albada et al. ${ }^{20}$ In short, 40 randomly chosen pulmonary intra-acinar vessels $<50 \mu \mathrm{m}$ were assessed at $400 \times$ magnification using an image analysis system (CZ KS400; Imaging Associates, Bicester, UK). Two different vascular areas were defined: outer vessel area and luminal area. The outer vessel area was defined as the area within the external elastic lamina. The area within the internal elastic lamina was defined as the luminal area. Areas were transformed into diameters and subsequently wall thickness was defined by subtracting the external diameter from the luminal diameter. Occlusion was then calculated in these pulmonary vessels accordingly, as (outer vessel diameter-luminal diameter)/(outer vessel diameter). Vessels were excluded from the measurement if they were too elliptical (ratio of the largest and smallest diameter exceeded 2), had an incomplete circular shape, were collapsed along more than one-quarter of the vessel wall or were located adjacent to an airway.

\section{Haemodynamics}

To assess the effect of the early radiation response of the lung vasculature on pulmonary/cardiac haemodynamics, right ventricle and pulmonary artery pressure (RVP and PAP) were directly measured at the peak of pulmonary dysfunction, 8 weeks after lung irradiation, according to Rabinovitch et al. ${ }^{21}$ In short, the rats ( $\mathrm{n}=4$ per group) first were anaesthetised (isoflurane) and ventilated with room air. A fluid-filled catheter with the pressure transducer was induced via the right external jugular vein into the right ventricle (RV) cavity and guided to the pulmonary artery (PA) under pressure waveform monitoring to record the RVP and PAP. After completion of the haemodynamic measurement, the thorax was opened and heart and lungs were excised. The heart was divided into atria, ventricles and septum and weighted separately. Right ventricle hypertrophy (RVH) was assessed by measuring the ratio of the weights of RV to the combination of left ventricle and intraventricular septum (IVS). The measurements were compared with non-irradiated controls $(n=3)$.

\section{Breathing rate assay}

In preclinical studies, breathing rate (BR) is considered as a surrogate for pulmonary function. ${ }^{17} 22$ BR was measured before and every 2 weeks after irradiation up to week 10 ( $n=24$ per group), as previously described. ${ }^{17}$ The increase in the BR at this time, relative to the mean BR in weeks $0-2$ after irradiation, was used as an indicator of early pulmonary dysfunction. The BR of non-irradiated controls was available in our database from previous experiments $(n=9)$

\section{Statistics}

To test whether differences exist between groups in pressure measurements and RVH assessment, the post hoc Bonferroni test was performed using one-way analysis if variance. For morphology quantification, the linear mixed model and F-test were specifically used to test differences among quantified parameters when comparing all groups at once. In this test randomly chosen vessels per rat per group were considered as
Figure 1 Overview of applied irradiation ports.

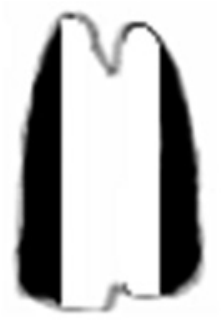

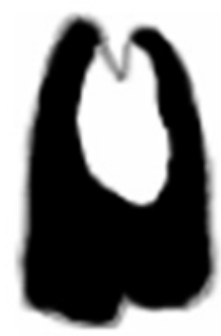

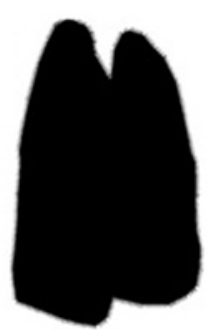


Figure 2 Global loss of pulmonary endothelial cells (ECs) precedes parenchymal damage. The effect of lung irradiation on lung tissue and ECs was assessed by evaluation of the morphology of lung tissue by haematoxylin and eosin, and immunohistochemical staining of rat EC specific marker (HIS52) respectively. Lung samples were taken from both in-field and out-of-field 2 weeks after $50 \%$ lung irradiation at $20 \mathrm{~Gy}$. (A) A large and small vessel in non-irradiated lung tissue with fine lung parenchyma. A close view of a small vessel in nonirradiated lung tissue shows an intact endothelial layer; the integrated lining of ECs is shown at a higher magnification. Perivascular oedema (in-field and outof-field, B and C, respectively; pointed by arrows) and EC detachment (leading to a disrupted endothelial layer) are the predominant damage. The results are representative sections from three irradiated rat lungs.
A
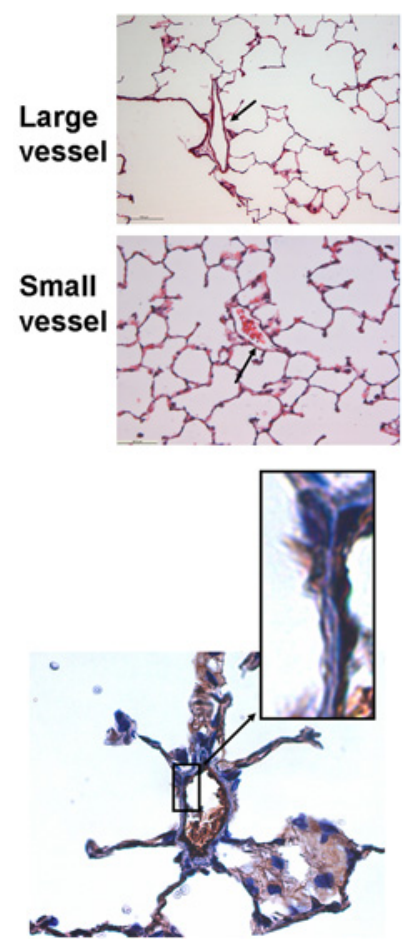

B
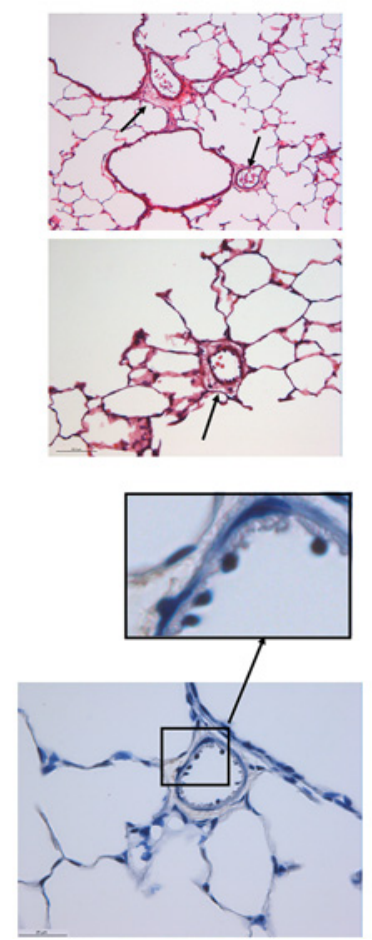

C
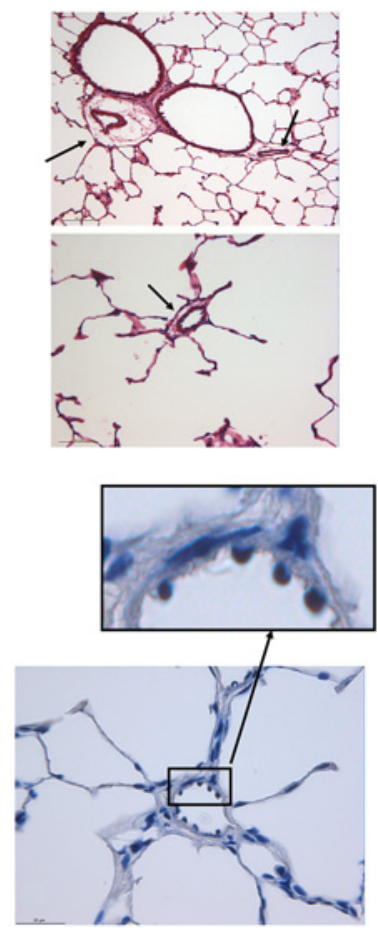

randomly repeated variables. The nominal level of significance was 0.05 .

To determine to what extent the changes in BR are explained by changes in PAP, Pearson's product-moment correlation coefficient of these two parameters was used. A correlation was considered significant if the hypothesis of no correlation was rejected at $\mathrm{p}<0.05$. BR varies significantly due to random errors in the measurement. Therefore when using all individual data points for correlation, to answer our main question, the correlation coefficient has to be corrected for attenuation due to the random errors of $\mathrm{BR}$ measurement according to the following formula: ${ }^{23}$

$$
r_{\text {corrected }}=\frac{r_{m P A P, B R}}{\sqrt{\text { reliability } B R}}
$$

The reliability is inversely related to random errors and is the consistency of a set of measurements. ${ }^{24}$ To assess the random error in the BR measurement, four random groups of rats with random dose distributions were chosen to resemble the variation in the irradiated population. Their BR measurements during the whole follow-up post irradiation were duplicated, and carried out by the same person. The reliability was then estimated as the concordance correlation coefficient between these two administrations of the same measure ${ }^{24}$ and was $0.77 .^{25}$

\section{RESULTS}

\section{Global loss of pulmonary endothelial cells precedes parenchymal damage and induces global pulmonary vascular remodelling}

At 2 weeks after $50 \%$ lung irradiation to $20 \mathrm{~Gy}$, prior to pulmonary dysfunction, ${ }^{17}$ the morphology of irradiated and shielded lung tissue was evaluated. In previous work we showed that this irradiation setting results in a comparable level of parenchymal and vascular damage at 8 weeks post irradiation, the peak of pulmonary dysfunction. ${ }^{7}{ }^{17}$ Indeed, prominent perivascular oedema in large and small vessels was observed in the radiation field (in-field), whereas besides minor changes in the epithelium, no major morphological changes could be observed in the alveolar parenchyma (figure 2A,B). Interestingly, a similar effect was observed in the shielded part of the lung (out-of-field) which received no dose, suggesting a vascular effect throughout the entire lung (figure 2C).

Besides the vascular oedema, loss of cells expressing the rat EC-specific marker HIS52 was observed in-field and out-of-field (figure 2A-C), indicating a global detachment of ECs and disruption of the endothelium in pulmonary vasculature. Since it is well known that changes in the integrity of ECs lead to increased permeability and leakage in irradiated tissues, ${ }^{26}$ the early response of pulmonary vasculature to lung irradiation may be initiated from the global lung EC loss. EC injury is a hallmark in initiating/mediating structural changes in pulmonary vasculature. $^{27}$ Therefore, the morphology of the lung vasculature was investigated at the peak of pulmonary dysfunction. ${ }^{17}$ Lung irradiation-induced structural changes in the vasculature, such as muscularisation of the media layer, thickening of the adventitia and more advanced lesions, including neointimal lesions and obliteration of small pulmonary vessels (figure $3 \mathrm{~B}$ ), were indicative of advanced remodelling of all layers. As expected, due to the global nature of vascular response, these features were also present out-of-field. To check whether the vascular response occurs at lower doses, the morphology of the lungs was evaluated after irradiation of the whole lung with 13 Gy. In previous work ${ }^{7} 17$ we showed that this irradiation setting has the lowest applicable dose which produces a pronounced pulmonary dysfunction. Prior to pulmonary dysfunction, predominant perivascular oedema was observed with no sign of parenchymal damage (see online supplementary figure $1 \mathrm{~A}$ ). At the peak of pulmonary dysfunction again severe vascular remodelling was observed without major parenchymal remodelling (see online supplementary figure $1 \mathrm{~B}$ ). This may 
Figure 3 Early radiation response of lung vasculature leads to global pulmonary vascular remodelling. (A) Anatomy of non-irradiated control pulmonary intra-acinar vessel $(<50 \mu \mathrm{m})$. Pulmonary vascular remodelling was assessed by immunohistochemical staining (Verhoeff's elastica stain) of pulmonary intra-acinar vessels 8 weeks post irradiation of 33,50 and $75 \%$ of rats' lungs to 28,22 and $17 \mathrm{~Gy}$, respectively. (B) Lung irradiation induces vascular remodelling such as muscularisation adventitia thickening and neointima formation. (C) Vascular remodelling was present in-field and out-of-field showing a global pulmonary vascular response in all irradiated volumes. $\mathrm{L}$, luminal diameter; 0 , outer vessel diameter; SMC, smooth muscle cell. Magnification $400 \times$.
A

B
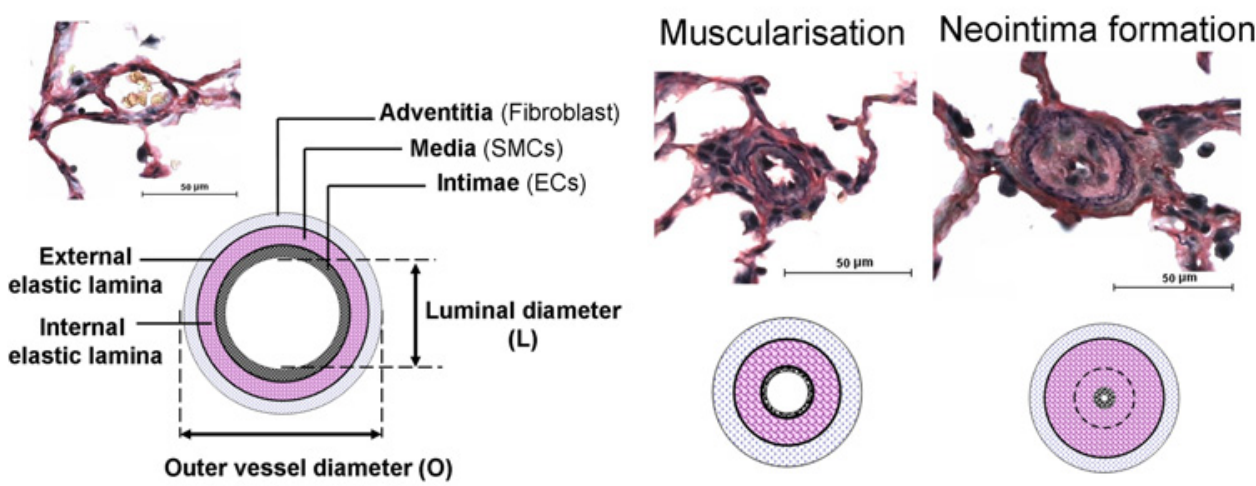

C
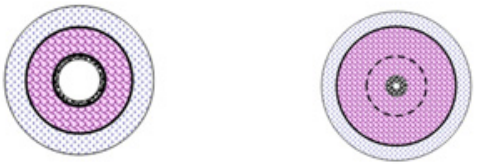
33\%, 28 Gy $\quad 50 \%, 22$ Gy $\quad 75 \%, 17$ Gy
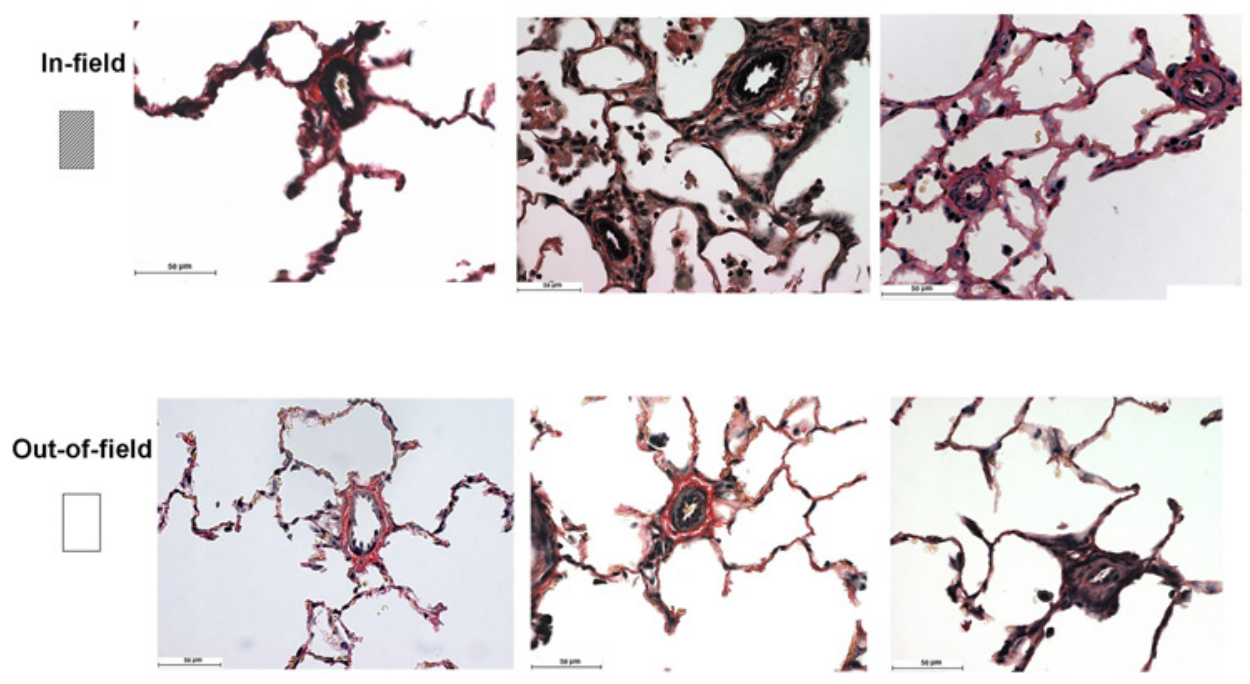

indicate that the tolerance dose for vascular response is lower than the parenchymal response.

\section{Global response of pulmonary vasculature is dependent on irradiated volume}

Development of pulmonary dysfunction depends on the irradiated lung volume. 1728 Therefore, the extent to which pulmonary dysfunction is determined by pulmonary vascular remodelling was assessed by morphological quantification of vascular damage, induced to different levels by irradiating different lung volumes (figure 3C) Strikingly irradiated-volume dependent increases were observed in absolute wall thickness (figure $4 \mathrm{~B}, \mathrm{p}<0.05$ ) and the percentage of luminal occlusion (figure $4 \mathrm{C}, \mathrm{p}<0.05$ ). Vascular remodelling was more pronounced with an increase in irradiated volume despite the lower radiation dose. Moreover, the out-of-field response increased as the irradiated volume increased (figure $4 \mathrm{~B}, \mathrm{C}, \mathrm{p}<0.05$ ). This indicates a global pulmonary vascular response rather than a response confined only to the area where the radiation dose is deposited.

\section{Global pulmonary vasculature response determines pulmonary dysfunction}

Global changes in vascular bed increase the pulmonary vascular resistance and induce elevated RVP and PAP, features that are known as pulmonary hypertension. ${ }^{29}$ Indeed lung irradiation was found to induce pulmonary hypertension by showing an irradiated-volume dependent increase in all components of RVP and PAP, despite the decreasing dose (figure 5A). Moreover, as can be expected with an increase in pulmonary vascular resistance and PAP, the increased RV workload led to an irradiatedvolume dependent RVH and a decrease in IVS weight (figure $5 \mathrm{~B}$ ). These are generally considered as characteristics of the severity of pulmonary hypertension. ${ }^{29}$ Therefore the results showed that rats irradiated to the largest lung volume $(75 \%)$ even to a relatively low dose (17 Gy) suffer from severe pulmonary hypertension. In addition, as a measure of pulmonary function, the BR of the irradiated animals increased in a similar manner to all other measured parameters, indicating that the development of pulmonary dysfunction is related to pulmonary hypertension (figure 5C). To determine to what extent the global pulmonary vascular response and hence pulmonary hypertension are responsible for pulmonary dysfunction, the increase in $\mathrm{BR}$ and mean PAP were correlated using all individual data (figure 5C). Remarkably, a strong correlation of these two parameters (figure $5 \mathrm{C}, \mathrm{r}=0.9$ ) was found. Taken together, our results indicate that the global pulmonary vascular response plays a major role in the development of early radiation-induced pulmonary dysfunction. 
Figure 4 Early radiation response of lung vasculature depends on irradiated volume rather than on local dose and is a global effect in the lung. (A) An example of quantification of vascular response in lung tissue in-field and outof-field from different irradiated volumes 8 weeks post irradiation. A large overview of the lung tissue was shown with arrows pointing at remodelled pulmonary intra-acinar vessels $(<50 \mu \mathrm{m})$. Two vessels were chosen to show the procedure of quantification by measuring the absolute wall thickness and the percentage of luminal occlusion. This quantification was applied for 40 pulmonary intra-acinar vessels per rat per field (in-field or outof-field) and the average of the obtained values was used as an assessment of the vascular response of that particular lung tissue. $(B, C)$ Early pulmonary vascular response depends on irradiated lung volume and is a global effect in the lungs. For each group three rats and for each rat two fields (in-field or out-of-field) and for each field 40 randomly chosen vessels were used for the morphological quantification. The outcome from each group was subtracted from the outcome of the control group and then represented in the graphs. The data are presented as mean \pm SEM. A significant difference between groups was found at $p$ value $<0.0000001$. L, luminal diameter; 0 , outer vessel diameter.
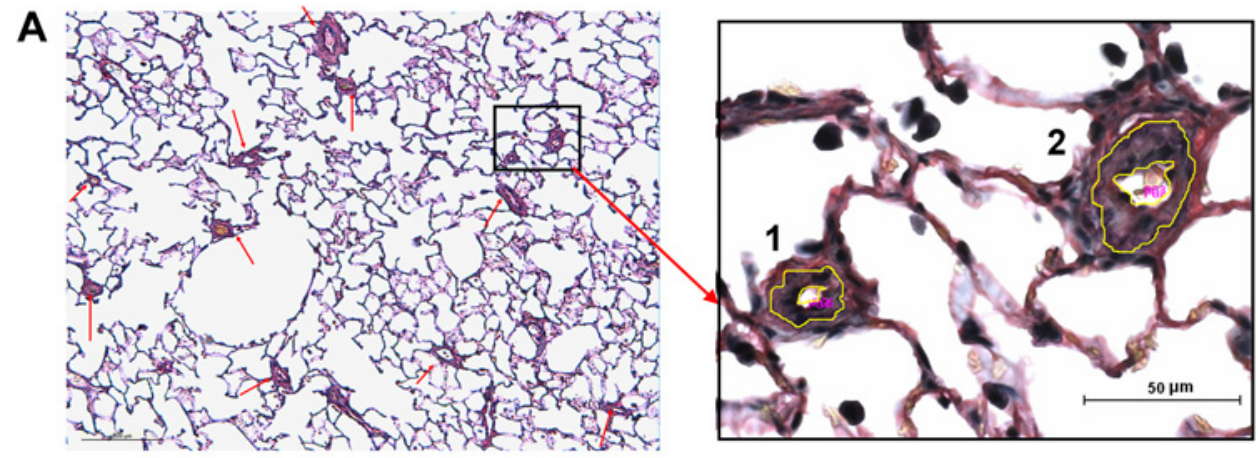

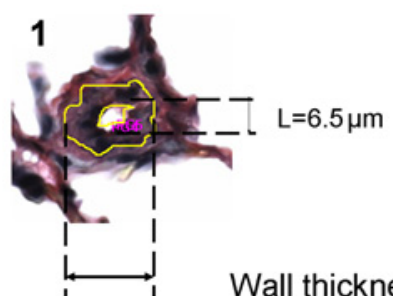

$\mathrm{O}=21.11 \mu \mathrm{m} \quad(\mathrm{O}-\mathrm{L}) / 2=7.3 \mu \mathrm{m}$

Luminal occlusion $=$ $((\mathrm{O}-\mathrm{L}) / \mathrm{O}) \times 100=69.2 \%$

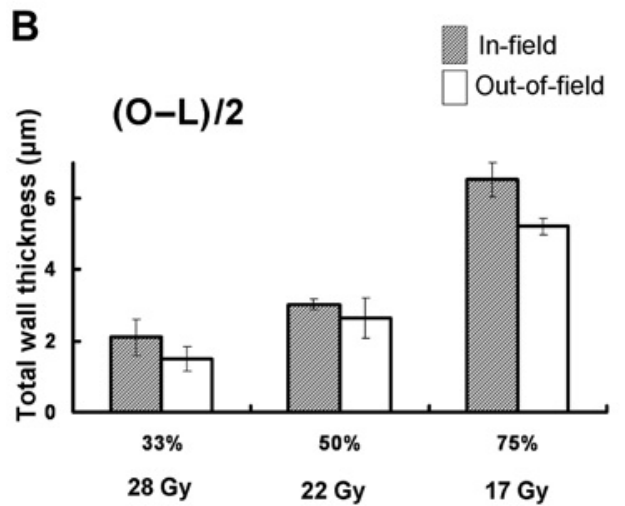

2

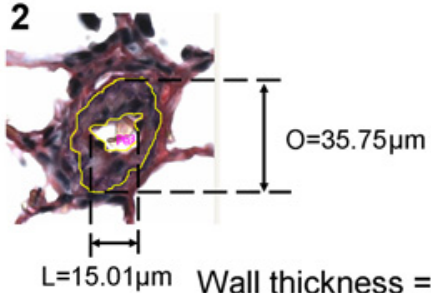

(O-L) $/ 2=10.37 \mu \mathrm{m}$

Luminal occlusion $=$

$((\mathrm{O}-\mathrm{L}) / \mathrm{O}) \times 100=58.03 \%$

C $((O-L) / O) \times 100$

In-field Out-of-field

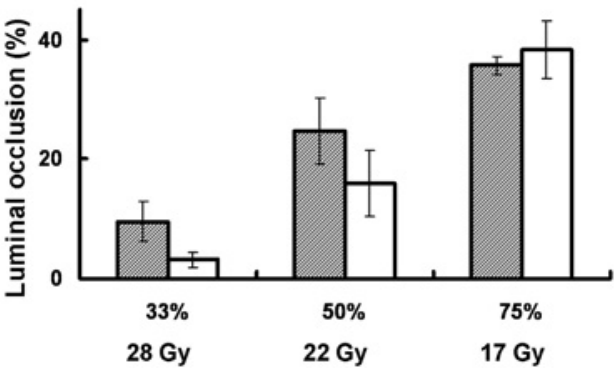

\section{DISCUSSION}

In preclinical studies pulmonary dysfunction after irradiation of large lung volumes seems to be caused by vascular damage. Since vascular remodelling is a hallmark of $\mathrm{PAH}$, we investigated whether irradiation could induce pulmonary hypertension with the related pulmonary vascular changes.

We show the proof of principle that irradiation of the lung leads to pulmonary vascular remodelling with subsequent pulmonary hypertension and RVH. The pulmonary vascular remodelling induced by irradiation showed striking similarities with the characteristic histopathology of PAH, including EC damage, vascular cell proliferation and neointimal lesions. ${ }^{30}$ The first signs of vascular changes such as EC loss and perivascular oedema were observed in the irradiated and shielded parts of the lung before any apparent parenchymal or BR changes. Subsequent to global pulmonary vascular remodelling accompanied by PAP elevation, RVH was observed, which is indicative of pulmonary hypertension. This cascade of events was shown to play a major role in the development of BR increase as a surrogate measure for pulmonary dysfunction.

$\mathrm{BR}$ is a non-specific measure for pulmonary function. It is only part of the lung function (which is not easily measurable in rats) and changes can be induced by several factors, such as reduced diffusion capacity due to parenchymal damage. However, the very strong correlation between pulmonary hypertension and $\mathrm{BR}$ in our study suggests that in our model the reduced ventilatory efficiency as a consequence of reduced perfusion is responsible for the increases in $\mathrm{BR}$, as frequently observed in patients with PAH. ${ }^{31}$ Hence in this model of PAH in rats, BR may seem to be a sufficient surrogate measure of pulmonary function.

The acute EC loss and perivascular oedema found in the current study may result from ECs detaching from the basal lamina, ${ }^{32}$ loss of endothelium integrity due to EC retraction and increased permeability to low molecular weight solutes ${ }^{26}$ shown to occur hours to days after radiation of pulmonary ECs. Although not yet studied in lungs, the importance of acute EC loss in the development of radiation-induced normal tissue damage has been established for other tissues such as intestine, spinal cord and rectum. ${ }^{33-35}$ In patients treated for rectal cancer with preoperative radiotherapy the radiation injury to normal tissue correlated strongly with the radiation-induced vascular damage as quantified by vascular wall thickness. ${ }^{33}$ The same study ${ }^{33}$ also demonstrated that irradiated ECs induce migration and proliferation of vascular smooth muscle cells, an important feature of vascular remodelling in $\mathrm{PAH}^{36}$ It therefore seems that ECs may be the main target of radiation in many organs and may have a pivotal role in the development of early radiation-induced vascular changes in the lung. However, the current study shows for the first time that this phenomenon can induce pulmonary hypertension and function failure. Indeed in many pulmonary vascular 
Figure 5 Early global response of pulmonary vasculature and the consequent pulmonary hypertension determines radiation-induced pulmonary dysfunction. (A) Pulmonary and cardiac haemodynamics measurement with right-sided cardiac catheterisation at 8 weeks post irradiation showed that all components of right ventricle pressure (RVP) and pulmonary artery pressure (PAP) increase with increasing irradiated volume. s, systolic; d, diastolic; m, mean. (B) The increased workload of the right ventricle (RV) led to irradiated volume-dependent increase in right ventricular hypertrophy (RVH). Left ventricle (LV), RV and intraventricular septum (IVS) weights of all groups are indicated in the table. (C) Early radiation-induced pulmonary dysfunction showed a similar dependence on irradiated volume as assessed by breathing rate (BR) increase. The strong correlation between increase in BR and mean PAP as a readout of global pulmonary vascular remodelling $\left(r_{\text {corrected }}=0.9\right)$ indicates that the development of pulmonary dysfunction strongly depends on the global response of lung vasculature. The increases in RVP, PAP and RVH of the irradiated rats were compared with control levels. The outcome for each group was subtracted from the outcome of the control group and then represented in graphs. Data are represented as mean \pm SEM. A significant difference in measurements of systolic RVP (sRVP), systolic PAP (sPAP), diastolic PAP (dPAP), mean PAP (mPAP), RVH and BR increase among the groups was found, with $p$ values of $0.004,0.0004,0.0001$, $0.00005,0.00006$ and 0.000003 , respectively.
A
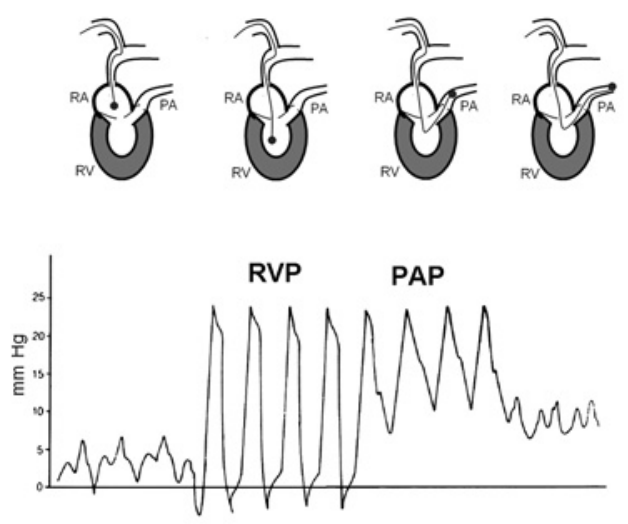

B

\begin{tabular}{|l|l|l|l|}
\hline Groups & $\begin{array}{l}\text { LV weight } \\
\text { (gr) }\end{array}$ & $\begin{array}{l}\text { RV weight } \\
\text { (gr) }\end{array}$ & $\begin{array}{l}\text { IVS weight } \\
\text { (gr) }\end{array}$ \\
\hline Con. & $0.6 \pm 0.01$ & $0.25 \pm 0.01$ & $0.24 \pm 0.01$ \\
\hline $33 \%$ & $0.55 \pm 0.02$ & $0.28 \pm 0.01$ & $0.22 \pm 0.01$ \\
\hline $50 \%$ & $0.58 \pm 0.02$ & $0.35 \pm 0.03$ & $0.17 \pm 0.008$ \\
\hline $75 \%$ & $0.64 \pm 0.008$ & $0.46 \pm 0.01$ & $0.15 \pm 0.02$ \\
\hline
\end{tabular}

C

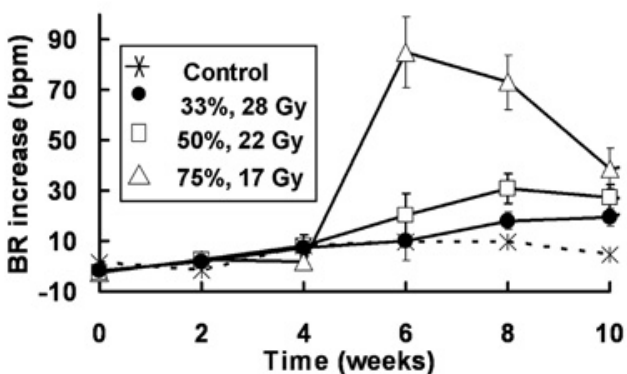

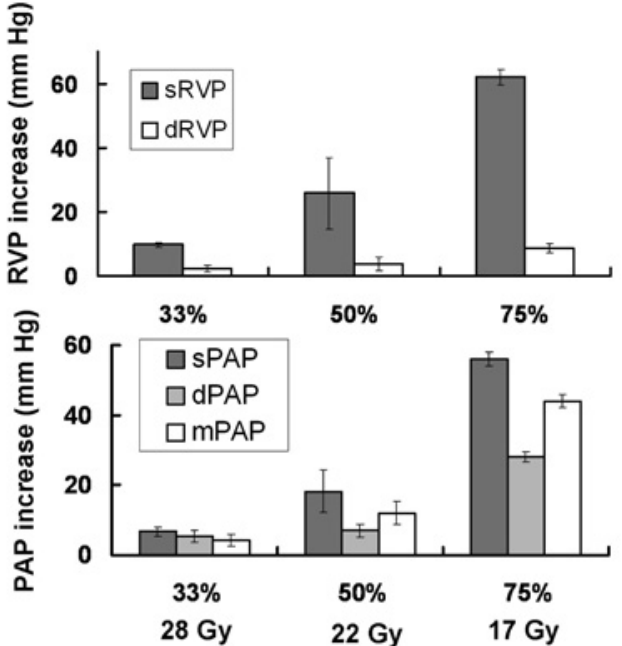
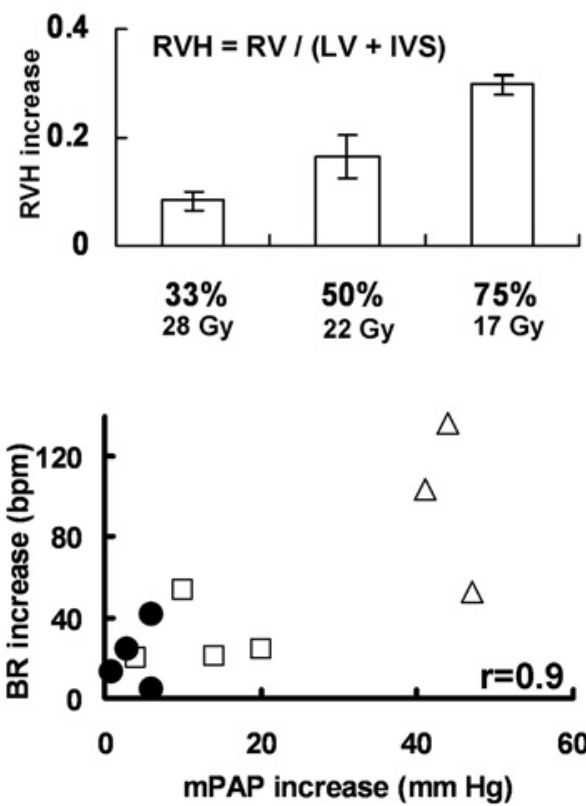

diseases, such as PAH, EC dysfunction is considered to play an initiating role in the pathogenesis of vascular remodelling. ${ }^{27}$ The features of vascular remodelling in our model show striking similarities with those observed in other experimental pulmonary hypertension models using the same strain of rats of the same age, ${ }^{20}$ or Sprague Dawley rats with almost the same age and weight $(300-350 \mathrm{~g}) .{ }^{37} 38$ This supports the hypothesis that EC loss also plays an initiating role in the pathogenesis of the early radiation-induced vascular changes in the lung. Moreover, in other animal models observations in line with ours have been published after thoracic irradiation, such as acute vascular remodelling in August rats ${ }^{39}$ and increased mean pulmonary artery pressure in Sprague Dawley rats, ${ }^{40}$ dogs ${ }^{41}$ and sheep. ${ }^{42} 43$ Therefore, our findings have opened up a new way of studying the radiation model of pulmonary hypertension to better understand EC dysfunction and degeneration of vasculature in pulmonary vascular disease leading to $\mathrm{PAH}$, a disease in which the inducing mechanisms are not completely understood. ${ }^{44}$

One of the most striking observations in this model of radiation-induced pulmonary vascular damage is that the histomorphology shows the development of neointimal lesions, which are considered hallmarks of PAH. ${ }^{6} 2046-49$ Treatment of patients with $\mathrm{PAH}$, in the international classification type I $\mathrm{PAH}$, still have a poor prognosis despite improvement in therapeutic regimens, ${ }^{1}$ hence it is referred to as the 'irreversible' form of $\mathrm{PAH}^{46}{ }^{47}$ Irreversibility of pulmonary hypertensive disease has been specifically associated with the presence of these neointimal lesions ${ }^{50}$ Although many PAH models have been shown to develop medial hypertrophy, few models have been described that consistently develop neointimal lesions. 629374951 In the current era, neointimal models are needed to adequately study the pathobiology of PAH and the effects of new treatment strategies. The occurrence of actual neointimal lesions in this radiation-induced pulmonary hypertension may offer such a novel model. A unique feature of the radiation-induced model is the dependence of irradiated lung volume on the induction of damage ${ }^{52}$ and consequent changes in PAP (present work), which facilitates well controlled induction to predetermined levels of $\mathrm{PAH}$ to study different stages of disease development in a controlled manner.

Radiation-induced EC loss (eg, due to apoptosis) and the consequent disruption of endothelial lining with increased permeability and perivascular oedema shown in this model may decrease the blood flow in the irradiated vasculature. As 
a compensatory effect, the pressure, blood flow and thereby the shear stress would increase in the vasculature in the non-irradiated part of the lungs. This increase of the shear stress may then lead to changes in function and structure of ECs in the non-irradiated vasculature. Indeed, changes in blood flow and shear stress are known to specifically regulate vascular remodelling by altering EC and smooth muscle cell apoptosis and proliferation rates. ${ }^{53} 54$ Increased shear stress induced by increased pulmonary blood flow in patients and animal models with large congenital cardiac shunts is known to lead to pulmonary endothelial dysfunction and progressive vascular remodelling and, thus, flow-associated $\mathrm{PAH} .{ }^{30} 44$ This may be a mechanism through which out-of-field EC loss and whole lung vascular remodelling develops. It is expected that irradiation of larger lung volumes even at a low dose (high enough to induce EC apoptosis) induces a more pronounced increase in blood flow and shear stress in non-irradiated vasculatures, leading to an enhanced out-of-field effect. The unique behaviour of our radiation-induced pulmonary hypertension model enables different levels of vascular response to be induced in different parts of the lung and hence may provide opportunities to study the mechanisms involved in progressive vascular remodelling in flow-associated PAH.

So far, treatment for PAH has focused on the use of epoprostenol, phosphodiesterase inhibitors and endothelin receptor antagonists with limited success. ${ }^{1}$ Recently, the use of antiproliferative agents such as imatinib, a platelet-derived growth factor inhibitor, was suggested to be a new class of drugs leading to improved outcome of patients with $\mathrm{PAH}^{55}$ Alternatively, cell-based therapies such as the use of bone-marrow-derived endothelial progenitor cells have recently been introduced in experimental PAH and been shown to have a positive effect on pulmonary vascular haemodynamics. ${ }^{27} 5657$ For all putative new treatment strategies, however, it is of paramount importance that they show improvements in experimental models mimicking the unique histomorphology of $\mathrm{PAH}$, that is, with neointimal lesions.

\section{CONCLUSION}

Lung irradiation was shown to induce pulmonary vascular degeneration and pulmonary hypertension possibly initiated from EC loss. As the histopathology of these lesions closely resembles vascular remodelling in $\mathrm{PAH}$, including neointimal lesions, partial lung irradiation may be a promising new model to study and develop strategies for the prevention and treatment of PAH.

Funding This work was funded by the Dutch Cancer Society (grant no. 2007-3890) and the Innovational Research Incentives Scheme of the Netherlands Organisation for Scientific Research (NWO) (grant no. 916.76.029).

\section{Competing interests None.}

Contributors Designed the study: P van Luijk, R P Coppes, G Ghobadi; collected the data: P van Luijk, G Ghobadi, B Bartelds, S J van der Veen, M G Dickinson; performed research: P van Luijk, G Ghobadi, B Bartelds, S J van der Veen, M G Dickinson; analysed the data: P van Luijk, G Ghobadi, B Bartelds, S J van der Veen; wrote the manuscript: P van Luijk, R P Coppes, G Ghobadi; revised the manuscript: P van Luijk, R P Coppes, G Ghobadi, B Bartelds, M G Dickinson, S Brandenburg, R M Berger, J A Langendijk; contributed new reagents or analytic tools: P van Luijk, R P Coppes, B Bartelds, S Brandenburg, R M Berger, J A Langendijk. RPC and PvL contributed equally.

Provenance and peer review Not commissioned; externally peer reviewed.

\section{REFERENCES}

1. Humbert $\mathbf{M}$, Sitbon 0 , Chaouat $A$, et al. Survival in patients with idiopathic, familial, and anorexigen-associated pulmonary arterial hypertension in the modern management era. Circulation 2010;122:156-63.
2. Wagenvoort CA. The pathology of primary pulmonary hypertension. J Pathol 1970;101:P1.

3. van Wolferen SA, Marcus JT, Boonstra A, et al. Prognostic value of right ventricular mass, volume, and function in idiopathic pulmonary arterial hypertension. Eur Heart $J$ 2007; $28: 1250-7$.

4. Champion HC, Michelakis ED, Hassoun PM. Comprehensive invasive and noninvasive approach to the right ventricle-pulmonary circulation unit: state of the art and clinical and research implications. Circulation 2009;120:992-1007.

5. Chan SY, Loscalzo J. Pathogenic mechanisms of pulmonary arterial hypertension. J Mol Cell Cardiol 2008;44:14-30.

6. Stenmark KR, Meyrick B, Galie N, et al. Animal models of pulmonary arterial hypertension: the hope for etiological discovery and pharmacological cure. Am J Physiol Lung Cell Mol Physiol 2009;297:L1013-32.

7. Novakova-Jiresova A, van Luijk $\mathrm{P}$, van Goor $\mathrm{H}$, et al. Changes in expression of injury after irradiation of increasing volumes in rat lung. Int $J$ Radiat Oncol Biol Phys 2007:67:1510-18.

8. Schallenkamp JM, Miller RC, Brinkmann DH, et al. Incidence of radiation pneumonitis after thoracic irradiation: dose-volume correlates. Int J Radiat Oncol Biol Phys 2007;67:410-16.

9. Semenenko VA, Molthen RC, Li C, et al. Irradiation of varying volumes of rat lung to same mean lung dose: a little to a lot or a lot to a little? Int J Radiat Oncol Biol Phys 2008;71:838-47.

10. Ghosh SN, Wu Q, Mader M, et al. Vascular injury after whole thoracic x-ray irradiation in the rat. Int J Radiat Oncol Biol Phys 2009;74:192-9.

11. Berger RM, Cromme-Dijkhuis AH, Hop WC, et al. Pulmonary arterial wall distensibility assessed by intravascular ultrasound in children with congenital heart disease: an indicator for pulmonary vascular disease? Chest 2002;122:549-57.

12. Murayama S, Akamine T, Sakai S, et al. Risk factor of radiation pneumonitis: assessment with velocity-encoded cine magnetic resonance imaging of pulmonary artery. J Comput Assist Tomogr 2004;28:204-8.

13. van Luijk $\mathbf{P}$, Faber $\mathrm{H}$, Meertens $\mathrm{H}$, et al. The impact of heart irradiation on dose-volume effects in the rat lung. Int J Radiat Oncol Biol Phys 2007;69:552-9.

14. van Luijk $\mathbf{P}$, Novakova-Jiresova $\mathrm{A}$, Faber $\mathrm{H}$, et al. Radiation damage to the heart enhances early radiation-induced lung function loss. Cancer Res 2005:65:6509-11.

15. Luijk P, Bijl HP, Coppes RP, et al. Techniques for precision irradiation of the lateral half of the rat cervical spinal cord using $150 \mathrm{MeV}$ protons. Phys Med Biol 2001;46:2857-71.

16. van Luijk $\mathbf{P}$, van $t^{\prime}$ Veld $A A$, Zelle $H D$, et al. Collimator scatter and $2 \mathrm{D}$ dosimetry in small proton beams. Phys Med Biol 2001;46:653-70.

17. Novakova-Jiresova $\mathbf{A}$, van Luijk $\mathrm{P}$, van Goor $\mathrm{H}$, et al. Pulmonary radiation injury: identification of risk factors associated with regional hypersensitivity. Cancer Res 2005; 65:3568-76.

18. O'Connor MM, Mayberg MR. Effects of radiation on cerebral vasculature: a review. Neurosurgery 2000;46:138-49; discussion 150-1.

19. Effros RM, Parker JC. Pulmonary vascular heterogeneity and the Starling hypothesis. Microvasc Res 2009:78:71-7.

20. van Albada ME, Schoemaker RG, Kemna MS, et al. The role of increased pulmonary blood flow in pulmonary arterial hypertension. Eur Respir J 2005;26:487-93.

21. Rabinovitch M, Gamble W, Nadas AS, et al. Rat pulmonary circulation after chronic hypoxia: hemodynamic and structural features. Am J Physiol 1979;236:H818-27.

22. Ward HE, Kemsley L, Davies L, et al. The pulmonary response to sublethal thoracic irradiation in the rat. Radiat Res 1993;136:15-21.

23. Spearman C. The proof and measurement of association between two things. Int $J$ Epidemiol 2010;39:1137-50.

24. Bland JM, Altman DG. Statistical methods for assessing agreement between two methods of clinical measurement. Lancet 1986;1:307-10

25. Ghobadi G, Hogeweg LE, Faber $\mathrm{H}$, et al. Quantifying local radiation-induced lung damage from computed tomography. Int J Radiat Oncol Biol Phys 2010;76:548-56.

26. Waters CM, Taylor JM, Molteni A, et al. Dose-response effects of radiation on the endothelial cells in culture. Radiat Res 1996;146:321-8.

27. Jurasz $\mathbf{P}$, Courtman $D$, Babaie $S$, et al. Role of apoptosis in pulmonary hypertension from experimental models to clinical trials. Pharmacol Ther 2010;126:1-8.

28. Rodrigues G, Lock M, D'Souza D, et al. Prediction of radiation pneumonitis by dose-volume histogram parameters in lung cancer-a systematic review. Radiother Oncol 2004:71:127-38.

29. van Albada ME, du Marchie Sarvaas GJ, Koster J, et al. Effects of erythropoietin on advanced pulmonary vascular remodelling. Eur Respir J 2008;31:126-34.

30. Dickinson MG, Bartelds B, Molema G, et al. Egr-1 expression during neointimal development in flow-associated pulmonary hypertension. Am J Pathol 2011:179:2199-209.

31. Sun XG, Hansen JE, Oudiz RJ, et al. Exercise pathophysiology in patients with primary pulmonary hypertension. Circulation 2001;104:429-35.

32. Maisin JR, Reyners H, de Reyners EG. Changes in the ultrastructure and the permeability of the capillaries after irradiation. Bibl Anat 1977;15 Pt 1:311-14.

33. Milliat $\mathbf{F}$, Francois A, Isoir $\mathbf{M}$, et al. Influence of endothelial cells on vascular smooth muscle cells phenotype after irradiation: implication in radiation-induced vascular damages. Am J Pathol 2006;169:1484-95.

34. Paris F, Fuks Z, Kang A, et al. Endothelial apoptosis as the primary lesion initiating intestinal radiation damage in mice. Science 2001;293:293-7.

35. Li Y0, Chen P, Haimovitz-Friedman A, et al. Endothelial apoptosis initiates acute blood-brain barrier disruption after ionizing radiation. Cancer Res 2003:63:5950-6.

36. Morrell NW, Adnot S, Archer SL, et al. Cellular and molecular basis of pulmonary arterial hypertension. J Am Coll Cardiol 2009;54(1 Suppl):S20-31. 
37. Okada K, Tanaka $\mathrm{Y}$, Bernstein $\mathrm{M}$, et al. Pulmonary hemodynamics modify the rat pulmonary artery response to injury. A neointimal model of pulmonary hypertension. Am J Pathol 1997;151:1019-25.

38. Abe K, Toba M, Alzoubi A, et al. Formation of plexiform lesions in experimenta severe pulmonary arterial hypertension. Circulation 2010;121:2747-54.

39. Down JD. The nature and relevance of late lung pathology following localised irradiation of the thorax in mice and rats. Br J Cancer Supp/ 1986;7:330-2.

40. Ward WF, Lin PJ, Wong PS, et al. Radiation pneumonitis in rats and its modification by the angiotensin-converting enzyme inhibitor captopril evaluated by high-resolution computed tomography. Radiat Res 1993;135:81-7.

41. Gillette SM, Powers BE, Orton EC, et al. Early radiation response of the canine heart and lung. Radiat Res 1991;125:34-40.

42. Loyd JE, Bolds JM, Sheller JR, et al. Acute effects of thoracic irradiation on lung function and structure in awake sheep. J Appl Physiol 1987;62:208-18.

43. Guerry-Force ML, Perkett EA, Brigham KL, et al. Early structural changes in sheep lung following thoracic irradiation. Radiat Res 1988;114:138-53.

44. van Albada ME, Bartelds $B$, Wijnberg $H$, et al. Gene expression profile in flowassociated pulmonary arterial hypertension with neointimal lesions. Am J Physiol Lung Cell Mol Physiol 2010;298:L483-91.

45. Rabinovitch M. Pathobiology of pulmonary hypertension. Annu Rev Pathol 2007;2:369-99

46. Sakao S, Tatsumi K, Voelkel NF. Reversible or irreversible remodeling in pulmonary arterial hypertension. Am J Respir Cell Mol Biol 2010;43:629-34.

47. Wagenvoort CA, Mooi WJ. Plexogenic arteriopathy. In: Munro Neville A, Walker F, Gottlieb LS, eds. Biopsy Pathology of the Pulmonary Vasculature. London: Chapman \& Hall Medical, 1989:56-113.

48. Taraseviciene-Stewart L, Kasahara Y, Alger L, et al. Inhibition of the VEGF receptor 2 combined with chronic hypoxia causes cell death-dependent pulmonary endothelial cell proliferation and severe pulmonary hypertension. FASEB J 2001;15:427-38.

49. White RJ, Meoli DF, Swarthout RF, et al. Plexiform-like lesions and increased tissue factor expression in a rat model of severe pulmonary arterial hypertension. Am J Physiol Lung Cell Mol Physiol 2007;293:L583-90.

50. Wagenvoort CA. Open lung biopsies in congenital heart disease for evaluation of pulmonary vascular disease. Predictive value with regard to corrective operability. Histopathology 1985;9:417-36

51. Ivy DD, McMurtry IF, Colvin $\mathrm{K}$, et al. Development of occlusive neointimal lesions in distal pulmonary arteries of endothelin B receptor-deficient rats: a new model of severe pulmonary arterial hypertension. Circulation 2005;111: 2988-96.

52. Coppes RP, Muijs CT, Faber $\mathrm{H}$, et al. Volume-Dependent expression of in-field and out-of-field effects in the proton-irradiated rat lung. Int $J$ Radiat Oncol Biol Phys 2011:81:262-9.

53. Macario DK, Entersz I, Abboud JP, et al. Inhibition of apoptosis prevents shear-induced detachment of endothelial cells. J Surg Res 2008:147:282-9.

54. Ueno H, Kanellakis P, Agrotis A, et al. Blood flow regulates the development of vascular hypertrophy, smooth muscle cell proliferation, and endothelial cell nitric oxide synthase in hypertension. Hypertension 2000;36:89-96.

55. Schermuly RT, Dony E, Ghofrani HA, et al. Reversal of experimental pulmonary hypertension by PDGF inhibition. J Clin Invest 2005;115:2811-21.

56. Diller GP, Thum T, Wilkins MR, et al. Endothelial progenitor cells in pulmonary arterial hypertension. Trends Cardiovasc Med 2010;20:22-9.

57. Zhao YD, Courtman DW, Deng Y, et al. Rescue of monocrotaline-induced pulmonary arterial hypertension using bone marrow-derived endothelial-like progenitor cells: efficacy of combined cell and eNOS gene therapy in established disease. Circ Res 2005:96:442-50.

\section{Journal club}

\section{Bronchoalveolar lavage-directed therapy does not affect prevalence of Pseudomonas aeruginosa infection and structural lung injury in children with cystic fibrosis}

Early Pseudomonas aeruginosa pulmonary infections lead to increased morbidity and mortality in children with cystic fibrosis (CF). As this diagnosis is difficult to ascertain in the nonexpectorating patient, the authors set out to determine if bronchoalveolar lavage (BAL)directed therapy during the first 5 years of life leads to better outcomes than standard practice (which is therapy based on clinical features and oropharyngeal cultures).

This multicentre, randomised, parallel-group trial recruited patients between 1999 and 2005 from eight CF centres in Australia and New Zealand. Of 267 eligible infants, 36\% were excluded. Patients were randomly assigned in a 1:1 ratio to receive either BAL-directed or standard therapy.

Primary outcomes at 5 years were prevalence of $P$ aeruginosa on BAL cultures and total CFCT score, a measure of pulmonary structural damage. Secondary outcomes were $z$ scores for weight, height and body mass index, pulmonary function parameters, CF-CT score components, exacerbation rate, number and duration of hospitalisations for non- $P$ aeruginosa pulmonary infections, number of episodes of $P$ aeruginosa infection per child-year and final BAL microbiology and inflammatory indices.

This study did not find any statistically significant differences between groups for any primary or secondary outcomes, except for a reduced length of hospital stay for non- $P$ aeruginosa respiratory-related admissions in the BAL-directed therapy group. While this may help determine clinical management, it is essential to note that the study was not powered for secondary outcomes, and CF-CT scores have yet to be validated as a clinical outcome measure.

- Wainwright CE, Vidmar S, Armstrong DS, et al; ACFBAL Study Investigators. Effect of bronchoalveolar lavage-directed therapy on Pseudomonas aeruginosa infection and structural lung injury in children with cystic fibrosis: a randomized trial. JAMA 2011:306:163-71.

\section{H Haja Mydin}

Correspondence to Dr Helmy Haja Mydin, Department of Respiratory Medicine, Aberdeen Royal Infirmary, Foresterhill, Aberdeen AB25 2ZN, UK; enidan@doctors.org.uk 